\title{
Prognostic value of three-dimensional echocardiographic right ventricular ejection fraction in patients with pulmonary arterial hypertension
}

\author{
Mitsushige Murata1, Toshimitsu Tsugu², Takashi Kawakami², Masaharu Kataoka², \\ Yugo Minakata², Jin Endo², Hikaru Tsuruta², Yuji Itabashi², Yuichiro Maekawa², \\ Mitsuru Murata ${ }^{1}$, Keiichi Fukuda ${ }^{2}$ \\ ${ }^{1}$ Department of Laboratory Medicine, Keio University, Tokyo, Japan \\ ${ }^{2}$ Department of Cardiology, School of Medicine, Keio University, Tokyo, Japan
}

Correspondence to: Mitsushige Murata, email: muratam@keio.jp

Keywords: right ventricular function, right ventricular ejection fraction, pulmonary arterial hypertension, three-dimensional echocardiography, prognosis

Received: September 20, $2016 \quad$ Accepted: November 08, 2016

Published: November 22, 2016

\section{ABSTRACT}

Background: Right ventricular (RV) function is an independent predictor of clinical outcomes in patients with pulmonary arterial hypertension (PAH). However, it remains controversial which RV parameter should be measured as an appropriate index for the treatment of PAH. The aim of this study was to identify the most useful parameter that correlates with hemodynamics and predicts clinical outcomes in PAH.

Results: Most of the clinical and echocardiographic RV parameters were significantly correlated with pulmonary vascular resistance (PVR) as well as mean pulmonary arterial pressure (MPAP). Among these, three dimensional right ventricular ejection fraction (3DRVEF) showed the strongest hemodynamic correlation, followed by 6-minute walk distance. Receiver operating characteristic analysis of association with cardiac events including death, hospitalization, and intervention revealed a greater area under the curve for 3DRVEF than for MPAP (0.78 vs. 0.74). KaplanMeier analysis showed that patients with 3DRVEF less than $38 \%$ had significantly shorter event-free survival than those with greater than $38 \%(P=0.0007)$. Finally, the Cox proportional hazards analysis revealed that 3DRVEF, but not MPAP, was an independent predictor of clinical events in PAH.

Materials and Methods: Eighty-six consecutive patients were enrolled in this study. RV hemodynamic parameters were measured by right heart catheterization (RHC). RV function was assessed using two-dimensional speckle-tracking echocardiography and three-dimensional transthoracic echocardiography (3DTTE) to evaluate RV free wall global strain (RVFS) and RVEF.

Conclusions: RVEF measured by 3DTTE could be a useful parameter for noninvasively assessing RV hemodynamics and predicting the clinical outcomes in PAH patients.

\section{INTRODUCTION}

Pulmonary arterial hypertension (PAH) is a progressive disease characterized by elevated pulmonary vascular resistance (PVR) and resulting in right ventricular (RV) failure and death [1]. Accumulating reports have implicated RV function as an independent predictor of prognosis in PAH [2-8]. The RV sensitivity to afterload is heightened compared to the left ventricle (LV) and increased RV afterload can dramatically reduce stroke volume, thus RV dilation might adapt to maintain cardiac output, leading to a decreased RV ejection fraction (RVEF). Indeed, PAH patients show impaired RV longitudinal systolic function, assessed by RV free wall 
strain, and RV dyssynchrony [9]. Thus, RV dysfunction could be attributable to many factors including RVEF, RV strain, and RV dyssynchrony, and in PAH, although such dysfunction may be induced mainly by the progressive elevation of PVR, the effects of increased afterload on RV remodeling could be diverse, even in the same degree of $\mathrm{PAH}$, probably due to different levels of contractile reserve and myocardial damage.

RVEF is the most commonly used index of RV contractility, and RVEF measured by cardiac magnetic resonance (CMR) might be useful for risk stratification and clinical management of patients with interstitial lung disease [6]. However, RVEF is highly dependent on loading conditions [10,11], and thus might not always adequately reflect contractility. In PAH, RVEF may be influenced by several factors including RV contractility, disease severity, and RV size. Regarding the RV contractility, RV strain might reflect the regional wall contraction more accurately and precisely than RVEF, although RV strain would be also affected by $\mathrm{RV}$ afterload (severity of $\mathrm{PAH}$ ). RV contraction is sequential, starting with contraction of the inlet and trabeculated myocardium and ending with contraction of the infundibulum. Thus, there is a slight regional time lag of contraction even under non-disease conditions. However, excessive RV dyssynchrony could potentially reduce cardiac output or increase filling pressure [12]. Thus, it remains controversial which of these RV parameters is the most useful to evaluate the mechanism of dysfunction and predict clinical outcomes in PAH. The aim of this study was therefore to evaluate such mechanisms in patients with $\mathrm{PAH}$ by comparing the echocardiographic parameters, assessed by speckle tracking echocardiography and three-dimensional transthoracic echocardiography (3DTTE), with hemodynamic parameters measured by right-sided heart catheterization (RHC).

\section{RESULTS}

The clinical characteristics for all subjects in the study $(n=86)$ are given in Table 1 . The majority of patients were female and middle aged, as consistent for PAH. The mPAP ranged from 13 to $68 \mathrm{mmHg}$ (median 31 $\mathrm{mmHg})$. Eight patients had normal mPAP $(<20 \mathrm{mmHg})$ and eleven had borderline mPAP $(20-24 \mathrm{mmHg})$, resulting in the relatively low mean mPAP of $35 \mathrm{mmHg}$. The pooled data for hemodynamic and echocardiographic parameters are summarized in Table 2. Hemodynamic parameters indicated an increased MPAP and PVR, while LV function was almost normal. The averaged values for echocardiographic RV parameters were borderline normal according to the ASE guideline [13], although RV fractional area change (RVFAC) and 3DRVEF were lower than the normal limits (Table 3 ).

\section{Correlation of RV function with hemodynamics}

RV systolic function, as assessed by several different echocardiographic parameters, was significantly correlated with hemodynamics, except for RAP (Table 4). Compared with conventional RV parameters such as Tricuspid annular plane systolic excursion (TAPSE), RVS', RV index of myocardial performance (RIMP), and RVFAC, RV strain measured by twodimensional speckle tracking echocardiography (2DSTE), and RV volumetric parameters measured by 3DTTE showed the highest correlation to hemodynamic parameters, with 3DRVEF the strongest parameter.

\section{Comparison of baseline characteristics of patients with and without clinical events}

The primary end point of pre-specified clinical events occurred in 19 of the 86 patients $(16 \%)$ as follows: 2 (5.9\%) deaths, 9 (9.8\%) hospitalizations, 1 pulmonary endoarterectomy (PEA), and 7 balloon pulmonary angioplasty (BPA) for deteriorating rightsided heart failure (Table 5). The group with clinical events were older and showed worse symptoms for RV hemodynamics and function than with the group without events, while LV function was comparable between the groups. RV contraction and RV dyssynchrony were also significantly impaired in the group with events, compared to that without events. Among the echocardiographic RV parameters, 3DRVEF showed the strongest difference between groups.

\section{Association of clinical, echocardiographic, and hemodynamic parameters with clinical events}

The univariate Cox proportional hazards analysis showed that most parameters were significantly associated with clinical events, while some of them including sex, LVEF, RVD, RV end-diastolic area index (RVEDAI), and RIMP had no correlation (Table 6). Multivariate Cox proportional hazards analysis revealed that 3DRVEF and 6MWD, but not mPAP, were independent predictors of clinical events. As shown in Figure 1A, The receiveroperating characteristic curve (ROC) analysis of association with clinical events identified a baseline mPAP of $35 \mathrm{mmHg}$ and a baseline 3DRVEF of $38 \%$ as the best cutoff values for predicting clinical events (mPAP: area under the curve 0.76 , sensitivity $79 \%$, specificity $69 \%$, $P$ value 0.0009 , 3DRVEF: area under the curve 0.78 , sensitivity $69 \%$, specificity $77 \%, P$ value 0.0004$)$. KaplanMeier analysis demonstrated that long-term outcomes for patients with $\mathrm{mPAP} \geqq 35 \mathrm{mmHg}$ were worse than for those with mPAP $<35 \mathrm{mmHg}$ (log-rank $P=0.0001$, Figure 1B). Furthermore, ROC analysis identified a 3DRVEF of $38 \%$ as the best cutoff value for predicting clinical events in patients with severe PAH (AUC 0.67, 


\begin{tabular}{lc}
\hline Subjects & 86 \\
Age, $\mathrm{y}$ & $50 \pm 17$ \\
Female, $n(\%)$ & $63(72)$ \\
Height, $\mathrm{m}$ & $1.59 \pm 0.58$ \\
Weight, $\mathrm{kg}$ & $58 \pm 12$ \\
BSA, ${ }^{2}$ & $1.6 \pm 0.2$ \\
WHO functional class & 16 \\
I & 41 \\
II & 29 \\
III & \\
Medications at baseline, $n(\%)$ & $60(70)$ \\
PDE-5I, $n(\%)$ & $51(59)$ \\
ERA, $n(\%)$ & $24(28)$ \\
Prostanoid, $n(\%)$ & $9(10)$ \\
CCB, $n(\%)$ & $20(23)$ \\
VKA, $n(\%)$ & $6.1 \pm 2.0$ \\
Uric acid, mg/dL & $100 \pm 200$ \\
BNP, pg/mL & $378 \pm 102$ \\
6 MWD, $m$ &
\end{tabular}

Unless indicated otherwise, data are given as the mean $\pm \mathrm{SD}$.

Abbreviations: WHO, World Health Organization; angioplasty; PDE-5I, Phosphodiesterase-5 inhibitors; ERA, Endothelin receptor antagonist; CCB, Calcium channel blockers; BNP, B-Type natriuretic peptide; 6MWD, 6-min walk distance.

sensitivity $46 \%$, specificity $84 \%, P$ value 0.06 ), and Kaplan-Meier analysis revealed that long-term outcomes for patients with $3 \mathrm{DRVEF}<38 \%$ were worse than for those with $3 \mathrm{DRVEF} \geqq 38 \%$ (log-rank $P=0.0002$, Figure 1C). Although long-term outcomes for patients with $\mathrm{mPAP} \geqq 35 \mathrm{mmHg}$ were poor, some of those patients with preserved 3DRVEF ( $\geqq 28 \%$ ) actually had a better prognosis (Figure 1D).

\section{DISCUSSION}

This study demonstrated that echocardiographic 3DRVEF significantly correlated with invasively measured hemodynamics and thus could be a noninvasive indicator of significant PAH hemodynamics. Furthermore, 3DRVEF was an independent and the strongest predictor of clinical events in patients associated with PAH, implicating the predominant significance of echocardiographic 3DRVEF compared with hemodynamic parameters including mPAP and PVR.

\section{Comparision of echocardiographic assessment with clinical and hemodynamic conditions}

As shown in previous studies, conventional RV parameters including TAPSE [14, 15], RVFAC [2], and RV S' [14] correlated with cardiac hemodynamics, albeit weakly. However, there are few reports that simultaneously compare the correlations of these parameters. The results presented herein comparatively analyzed both clinical, hemodynamic data and echocardiographic RV function including RV contraction and RV dyssynchrony, and confirmed the prognostic significance of WHO functional class, 6MWD, cardiac index, and most of the echocardiographic RV parameters. Importantly, univariate and multivariate Cox proportional hazard analysis identified echocardiographic 3DRVEF, but not mPAP, as an independent predictor of clinical events (Table 6). These results emphasize the predominant significance of RV function assessed by 3DRVEF, compared with hemodynamic parameters.

\section{Prognostic value of echocardiographic 3DRVEF in patients with $\mathrm{PAH}$}

Previous studies showed that mortality in $\mathrm{PAH}$ patients is associated with both the severity of symptoms and the extent of right heart failure, [16] and that RV function is a predictor of mortality in these patients, as evidenced by the correlation between clinical outcomes and several RV parameters including TAPSE, RV S', RVFAC, 
Table 2: Hemodynamic and echocardiographic parameters for all subjects

\begin{tabular}{|c|c|}
\hline \multicolumn{2}{|l|}{ Hemodynamics } \\
\hline Systolic blood pressure, $\mathrm{mmHg}$ & $111 \pm 17$ \\
\hline Diastolic blood pressure, $\mathrm{mmHg}$ & $64 \pm 13$ \\
\hline Heart rate, beats/min & $72 \pm 14$ \\
\hline Right atrial pressure, $\mathrm{mmHg}$ & $5.7 \pm 2.7$ \\
\hline mPAP, $\mathrm{mmHg}$ & $34 \pm 12$ \\
\hline PCWP, mmHg & $8.7 \pm 3.0$ \\
\hline PVR, dyne $\bullet \mathrm{sec} \cdot \mathrm{cm}^{-5}$ & $564 \pm 375$ \\
\hline Cardiac index, $\mathrm{L} / \mathrm{min}$ per $\mathrm{m}^{2}$ & $2.5 \pm 0.6$ \\
\hline \multicolumn{2}{|l|}{ Echocardiographic } \\
\hline LV end-diastolic diameter, $\mathrm{mm}$ & $44 \pm 5$ \\
\hline LV end-systolic diameter, mm & $26 \pm 5$ \\
\hline LV ejection fraction, $\%$ & $69 \pm 8$ \\
\hline $\mathrm{E}, \mathrm{cm} / \mathrm{s}$ & $69 \pm 19$ \\
\hline $\mathrm{A}, \mathrm{cm} / \mathrm{s}$ & $69 \pm 17$ \\
\hline E/A ratio & $1.1 \pm 0.4$ \\
\hline $\mathrm{E}^{‘}, \mathrm{~cm} / \mathrm{s}$ & $12.3 \pm 3.8$ \\
\hline $\mathrm{E} / \mathrm{E}^{\prime}$ ratio & $6.1 \pm 2.4$ \\
\hline IVC diameter, mm & $15 \pm 4$ \\
\hline Peak TR pressure gradient, $\mathrm{mmHg}$ & $52 \pm 20$ \\
\hline Right atrial area, $\mathrm{cm}^{2}$ & $18 \pm 5$ \\
\hline
\end{tabular}

Abbreviations: mPAP, mean pulmonary arterial pressure; PCWP, pulmonary capillary wedge pressure;

PVR, pulmonary vascular resistance; LV, left ventricular; IVC, inferior vena cava; TR, tricuspid regurgitation.

RV strain, and RVEF measured by CMR and 3DTTE $[17,5]$. Furthermore, Ryo et al. reported that 3DRVEF was a predictive parameter for association with the combined end point of hospitalization, death, or lung surgery [18]. Since RV systolic function is mediated by various factors such as RV contractility, RV synchronization, and PVR [1], the mechanisms for RV dysfunction should be identified for each pathophysiological condition. In the present study, we investigated all the echocardiographic and hemodynamic parameters associated with RV function, and found that among many RV parameters including RV strain, and RV dyssynchrony, 3DRVEF showed the strongest correlation with hemodynamics and prognosis. Historically, hemodynamic parameters including cardiac index and mPAP are used as prognostic predictors in PAH [19], with $\mathrm{RV}$ strain and RV dyssynchrony also used to predict clinical outcomes [17, 20]. However, consistent with our results, CMR studies demonstrated the prognostic importance of a decrease in RVEF $[8,21]$, which also accompanies late disease progression [22]. We postulate that 3DRVEF could be the most useful overall predictive parameter because it reflects both the cardiac systolic and hemodynamic functions associated with prognosis, since RVEF would be affected by RV contraction, RV dyssynchrony, and RV afterload $[1,23]$. Further studies are now clearly warranted to clarify the effect of RVEF on clinical outcomes in PAH.

\section{Clinical implications}

Although RHC is necessary for the diagnosis of pulmonary hypertension, repetitive RHC may not be applicable as a routine examination. The present study demonstrated the feasibility and convenience of noninvasive RV analysis using 3DTTE rather than RHC to evaluate the pathology in patients with $\mathrm{PAH}$. The diagnosis of PAH is defined as mPAP $>25 \mathrm{mmHg}$, which could be reliably detected by 3DRVEF measurments with a cutoff value of $45 \%$ (AUC 0.81 , sensitivity $60 \%$, specificity $92 \%, P=0.002$ ) and ePASP with a cutoff value of $36 \mathrm{mmHg}$ (AUC 0.82 , sensitivity $64 \%$, specificity $67 \%$, $P=0.001)$.

In the present study, 36 patients had severe PAH (mPAP $>35 \mathrm{mmHg}$ ) with a poor prognosis $(50 \%$ of event-free survival in 2.5 years) (Figure 1D). Notably, among this group, patients with $3 \mathrm{DRVEF} \geqq 28 \%$ had a better prognosis of $70 \%$ event-free survival in 2.5 years. 


\section{Table 3: Echocardiographic right ventricular parameters for all subjects}

\begin{tabular}{|c|c|}
\hline $\mathrm{RV}$ basal diameter, $\mathrm{mm}$ & $40.1 \pm 7.8$ \\
\hline $\mathrm{RV}$ mid cavity diameter, $\mathrm{mm}$ & $31.4 \pm 6.7$ \\
\hline RV longitudinal diameter, mm & $72.5 \pm 9.2$ \\
\hline $\mathrm{RV}$ end-diastolic area index, $\mathrm{cm}^{2} / \mathrm{m}^{2}$ & $10.1 \pm 3.3$ \\
\hline $\mathrm{RV}$ end-systolic area index, $\mathrm{cm}^{2} / \mathrm{m}^{2}$ & $15.9 \pm 5.7$ \\
\hline RV fractional area change, $\%$ & $31 \pm 11$ \\
\hline TAPSE, mm & $19 \pm 4$ \\
\hline $\mathrm{RV} \mathrm{S}^{\prime}, \mathrm{cm} / \mathrm{sec}$ & $11.4 \pm 3.1$ \\
\hline RV index of myocardial performance (tissue Doppler) & $0.52 \pm 0.24$ \\
\hline \multicolumn{2}{|l|}{ RV longitudinal strain } \\
\hline Base septal wall, \% & $-15.3 \pm 5.9$ \\
\hline Mid septal wall, $\%$ & $-17.2 \pm 6.4$ \\
\hline Apex septal wall, $\%$ & $-10.7 \pm 7.8$ \\
\hline Base free wall, $\%$ & $-21.6 \pm 9.6$ \\
\hline Mid free wall, \% & $-21.5 \pm 8.1$ \\
\hline Apex free wall, $\%$ & $-18.1 \pm 7.9$ \\
\hline Global , \% & $-16.7 \pm 4.8$ \\
\hline RVFS, $\%$ & $-19.7 \pm 6.4$ \\
\hline RV-SD6, ms & $80 \pm 36$ \\
\hline \multicolumn{2}{|l|}{$3 \mathrm{D}$} \\
\hline $\mathrm{RV}$ end-diastolic volume index, $\mathrm{ml}$ per $\mathrm{m}^{2}$ & $52 \pm 17$ \\
\hline $\mathrm{RV}$ end-systolic volume index, $\mathrm{ml}$ per $\mathrm{m}^{2}$ & $30 \pm 14$ \\
\hline RVEF, \% & $43 \pm 12$ \\
\hline
\end{tabular}

Abbreviations: RV; right ventricular; TAPSE, tricuspid annular plane systolic excursion; RVFS, RV free wall global strain; RVEF, right ventricular ejection fraction.

Table 4: Correlation of echocardiographic RV parameters with hemodynamics

\begin{tabular}{|c|c|c|c|c|c|c|c|c|c|c|c|c|c|c|}
\hline \multirow[t]{2}{*}{ RHC parameter } & \multicolumn{2}{|c|}{ TAPSE } & \multicolumn{2}{|c|}{ RV S' } & \multicolumn{2}{|c|}{ RIMP } & \multicolumn{2}{|c|}{ RVFAC } & \multicolumn{2}{|c|}{ RVFS } & \multicolumn{2}{|c|}{ 3DRVEF } & \multicolumn{2}{|c|}{ RV-SD6 } \\
\hline & $\mathrm{r}$ & $P$ & $\mathrm{r}$ & $P$ & $\mathrm{r}$ & $P$ & $\mathrm{r}$ & $P$ & $\mathrm{r}$ & $P$ & $\mathrm{r}$ & $P$ & $\mathrm{r}$ & $P$ \\
\hline Mean PAP, mmHg & -0.24 & 0.02 & -0.20 & 0.07 & 0.17 & 0.1 & -0.41 & $<0.0001$ & 0.51 & $<0.0001$ & -0.62 & $<0.0001$ & 0.46 & $<0.0001$ \\
\hline PVR, WU & -0.34 & 0.001 & -0.31 & 0.004 & 0.25 & 0.02 & -0.39 & 0.0002 & 0.60 & $<0.0001$ & -0.61 & $<0.0001$ & 0.44 & $<0.0001$ \\
\hline Mean RAP, mmHg & -0.11 & 0.3 & -0.16 & 0.1 & -0.09 & 0.4 & -0.20 & 0.06 & 0.14 & 0.2 & -0.31 & 0.005 & 0.16 & 0.1 \\
\hline $\mathrm{CI}, \mathrm{L} / \mathrm{min}$ per $\mathrm{m}^{2}$ & 0.31 & 0.003 & 0.43 & $<0.0001$ & -0.14 & 0.2 & -0.30 & 0.006 & -0.39 & 0.0004 & 0.26 & 0.02 & -0.23 & 0.03 \\
\hline
\end{tabular}

Abbreviations: RV indicates right ventricular; RHC, right heart catheterization; PAP, pulmonary arterial pressure; PVR, pulmonary vascular resistance; RAP, right atrial pressure; CI, cardiac index; TAPSE, tricuspid annular plane systolic excursion; RIMP, right ventricular index of myocardial performance; RVFAC, right ventricular fractional area change; RVFS, right ventricular free wall global strain; 3DRVEF, three-dimensional right ventricular ejection fraction.

Thus, risk stratification by 3DRVEF might identify PAH patients at higher risk of clinical deterioration. This is important, as timely intensification of PAH-specific therapy could prevent further clinical worsening and death.

\section{Study limitations}

This study was a single-center and retrospective cohort study with a small number of patients. Thus, the findings should be prospectively confirmed in a larger 
Table 5: Comparison of clinical, echocardiographic, and hemodynamic characteristics of the overall cohort based on clinical events

\begin{tabular}{|c|c|c|c|}
\hline & No event $(n=67)$ & Event $(n=19)$ & $P$ Value \\
\hline Age, $y$ & $48 \pm 17$ & $58 \pm 16$ & 0.02 \\
\hline Weight, kg & $57 \pm 10$ & $60 \pm 18$ & NS \\
\hline Height, cm & $158 \pm 9$ & $160 \pm 11$ & NS \\
\hline Female, $n(\%)$ & $52(78)$ & $12(63)$ & NS \\
\hline WHO & $2.0 \pm 0.7$ & $2.5 \pm 0.6$ & 0.009 \\
\hline $6 \mathrm{MWD}, \mathrm{m}$ & $400 \pm 85$ & $285 \pm 117$ & 0.0005 \\
\hline \multicolumn{4}{|l|}{ Hemodynamics } \\
\hline RAP, mmHg & $5.2 \pm 2.5$ & $7.5 \pm 2.6$ & 0.001 \\
\hline mPAP, $\mathrm{mmHg}$ & $32 \pm 12$ & $42 \pm 9$ & 0.0005 \\
\hline Cardiac index, $1 /$ min per $\mathrm{m}^{2}$ & $2.6 \pm 0.6$ & $2.2 \pm 0.5$ & 0.002 \\
\hline PVR, WU & $6.2 \pm 4.1$ & $10.2 \pm 5.3$ & 0.0005 \\
\hline \multicolumn{4}{|l|}{ Echocardiography } \\
\hline LVEF, \% & $68 \pm 8$ & $72 \pm 9$ & NS \\
\hline $\mathrm{E} / \mathrm{E}$ & $6.1 \pm 2.4$ & $6.2 \pm 2.4$ & NS \\
\hline $\mathrm{RA}$ area index, $\mathrm{mm}^{2} / \mathrm{m}^{2}$ & $11.2 \pm 2.7$ & $13.7 \pm 4.3$ & 0.01 \\
\hline $\mathrm{RV}$ basal diameter, $\mathrm{mm}$ & $39.2 \pm 7.3$ & $43.1 \pm 8.9$ & NS \\
\hline $\mathrm{RV}$ end-diastolic area index, $\mathrm{cm}^{2} / \mathrm{m}^{2}$ & $14.3 \pm 3.7$ & $15.3 \pm 4.9$ & NS \\
\hline $\mathrm{RV}$ end-systolic area index, $\mathrm{cm}^{2} / \mathrm{m}^{2}$ & $9.7 \pm 3.1$ & $11.5 \pm 3.8$ & 0.03 \\
\hline RV fractional area change, $\%$ & $33 \pm 10$ & $24 \pm 11$ & 0.003 \\
\hline TAPSE, mm & $19 \pm 4$ & $17 \pm 4$ & 0.04 \\
\hline $\mathrm{RV} \mathrm{S}^{\star}, \mathrm{cm} / \mathrm{sec}$ & $12.0 \pm 2.9$ & $9.4 \pm 2.9$ & 0.002 \\
\hline RV index of myocardial performance & $0.53 \pm 0.27$ & $0.53 \pm 0.27$ & NS \\
\hline \multicolumn{4}{|l|}{ Right ventricular longitudinal strain } \\
\hline Base septal wall, $\%$ & $-15.5 \pm 6.1$ & $-14.5 \pm 5.7$ & NS \\
\hline Mid septal wall, \% & $-17.6 \pm 6.6$ & $-15.7 \pm 5.9$ & NS \\
\hline Apex septal wall, $\%$ & $-11.7 \pm 7.0$ & $-7.2 \pm 9.2$ & 0.02 \\
\hline Base free wall, $\%$ & $-22.7 \pm 10.1$ & $-17.6 \pm 6.7$ & 0.04 \\
\hline Mid free wall, $\%$ & $-22.1 \pm 7.8$ & $-19.3 \pm 9.1$ & NS \\
\hline Mid free wall, $\%$ & $-18.8 \pm 6.9$ & $-15.7 \pm 10.3$ & NS \\
\hline Global, \% & $-17.6 \pm 4.3$ & $-14.0 \pm 5.8$ & 0.007 \\
\hline RVFS, \% & $-20.8 \pm 6.3$ & $-16.1 \pm 5.9$ & 0.004 \\
\hline RV-SD6, ms & $74 \pm 30$ & $101 \pm 47$ & 0.02 \\
\hline \multicolumn{4}{|l|}{$3 \mathrm{D}$} \\
\hline $\mathrm{RV}$ end-diastolic volume index, $\mathrm{ml} / \mathrm{m}^{2}$ & $49 \pm 16$ & $61 \pm 18$ & 0.02 \\
\hline $\mathrm{RV}$ end-systolic volume index, $\mathrm{ml} / \mathrm{m}^{2}$ & $28 \pm 13$ & $41 \pm 16$ & 0.0012 \\
\hline RVEF, \% & $46 \pm 12$ & $34 \pm 11$ & 0.0008 \\
\hline
\end{tabular}

Unless indicated otherwise, data are given as the mean \pm SD.

Abbreviations: WHO, World Health Organization; 6MWD, 6-minute walk distance; RAP, right atrial pressure; mPAP, mean pulmonary arterial pressure; PVR, pulmonary vascular resistance; RA, right atrial; RV, right ventricular; TAPSE, tricuspid annular plane systolic excursion; RVFS, right ventricular free wall global strain; 3DRVEF, three-dimensional right ventricular ejection fraction. 
Table 6: Univariate and multivariate cox proportional hazards analysis of the relationships with clinical events

\begin{tabular}{|c|c|c|c|}
\hline Variable & HR & $95 \% \mathrm{CI}$ & $P$ Value \\
\hline \multicolumn{4}{|l|}{ Univariate } \\
\hline Age & 1.04 & $1.01-1.07$ & 0.01 \\
\hline Sex & 1.54 & $0.54-3.96$ & NS \\
\hline WHO & 2.79 & $1.35-6.33$ & 0.005 \\
\hline 6MWD, m & 0.99 & $0.98-0.99$ & $<0.0001$ \\
\hline $\operatorname{logBNP}, \mathrm{pg} / \mathrm{mL}$ & 5.02 & $2.40-10.6$ & $<0.0001$ \\
\hline \multicolumn{4}{|l|}{ Hemodynamics } \\
\hline Mean PAP, mmHg & 1.06 & $1.03-1.10$ & 0.0009 \\
\hline RAP, mmHg & 1.28 & $1.09-1.49$ & 0.003 \\
\hline PVR, WU & 1.15 & $1.07-1.22$ & 0.0006 \\
\hline Cardiac index, $1 / \mathrm{min} / \mathrm{m}^{2}$ & 0.18 & $0.06-0.49$ & 0.0005 \\
\hline \multicolumn{4}{|l|}{ Echocardiography } \\
\hline $\mathrm{RA}$ area, $\mathrm{cm}^{2}$ & 1.20 & $1.06-1.34$ & 0.006 \\
\hline LV ejection fraction, $\%$ & 1.05 & $0.99-1.12$ & NS \\
\hline $\mathrm{RVD}, \mathrm{mm}$ & 1.70 & $0.99-2.70$ & NS \\
\hline $\mathrm{RV}$ end-diastolic area index, $\mathrm{cm}^{2} / \mathrm{m}^{2}$ & 1.08 & $0.97-1.19$ & NS \\
\hline $\mathrm{RV}$ end-systolic area index, $\mathrm{cm}^{2} / \mathrm{m}^{2}$ & 1.16 & $1.03-1.29$ & 0.02 \\
\hline RV fractional area change, $\%$ & 0.94 & $0.90-0.98$ & 0.002 \\
\hline TAPSE, mm & 0.85 & $0.74-0.98$ & 0.02 \\
\hline RVS', cm/s & 0.82 & $0.72-0.94$ & 0.004 \\
\hline $\mathrm{RV}$ index of myocardial performance & 1.21 & $0.12-4.26$ & NS \\
\hline $\mathrm{RV}$ end-diastolic volume index, $\mathrm{ml} / \mathrm{m}^{2}$ & 1.04 & $1.01-1.07$ & 0.02 \\
\hline $\mathrm{RV}$ end-systolic volume index, $\mathrm{ml} / \mathrm{m}^{2}$ & 1.06 & $1.02-1.09$ & 0.0008 \\
\hline 3DRVEF, \% & 0.92 & $0.88-0.96$ & 0.0001 \\
\hline RVFS, \% & 1.12 & $1.04-1.22$ & 0.003 \\
\hline RV-SD6, ms & 1.02 & $1.01-1.03$ & 0.002 \\
\hline \multicolumn{4}{|l|}{ Multivariate } \\
\hline 6MWD & 0.99 & $0.98-0.99$ & 0.03 \\
\hline mPAP & 0.98 & $0.90-1.04$ & NS \\
\hline 3DRVEF & 0.91 & $0.84-0.98$ & 0.01 \\
\hline
\end{tabular}

Abbreviations: CI, confidence interval; HR, hazard ratio; WHO, World Health Organization; 6MWD, 6-minute walk distance; RAP, right atrial pressure; mPAP, mean pulmonary arterial pressure; PVR, pulmonary vascular resistance; RA, right atrial; RV, right ventricular; TAPSE, tricuspid annular plane systolic excursion; 3DRVEF, three-dimensional right ventricular ejection fraction; RVFS, right ventricular free wall global strain.

population. In addition, subjects in our investigation were chosen as the patients who underwent RHC on suspicion of $\mathrm{PAH}$, implicating that the population included heterogeneous etiology that may weaken or modify the results.

\section{MATERIALS AND METHODS}

The ethics committee of Keio University Hospital approved this study, which comprised a retrospective analysis of patient records from a prospectively maintained registry of patients admitted to the Keio University Hospital. Of 198 consecutive patients who underwent RHC to diagnose pulmonary hypertension from September 2013 to December 2015, 134 patients assessed as eligible (68\%) underwent echocardiography including speckle tracking and three-dimensional echocardiography. Finally, 86 patients were included in the main outcome analysis, with 48 excluded due to congenital shunt disease 
(26), left-sided heart failure (4), or unanalyzable poor trace (18). Among 86 patients, 29 had idiopathic PAH, 6 heritable PAH, 17 collagen diseases, 4 pulmonary diseases, 22 chronic thromboembolic pulmonary hypertension (CTEPH), and 8 other etiologies including blood diseases.

\section{Right-sided heart catheterization}

All patients underwent RHC using a 6- or 7-Fr Swan-Ganz catheter (Swan-Ganz CCO CEDV; Edwards Life Sciences, Irvine, CA, USA). PAP, right atrial pressure (RAP), PCWP, and cardiac output (CO) were measured by RHC. CO was assessed using the thermodilution method or Fick technique, and cardiac index (CI) was calculated by dividing $\mathrm{CO}$ by body surface area. PVR was calculated using the follow formula: PVR $=(\mathrm{mPAP}-$ mean PCWP $) / \mathrm{CO}$.

\section{Echocardiographic measurements and analyses}

Patients underwent echocardiography using a Vivid-E9 ultrasound system (GE Healthcare, Horten, Norway). Established criteria were used to measure RV size and function [13]. RV end-diastolic area and endsystolic area were assessed using manual planimetry in

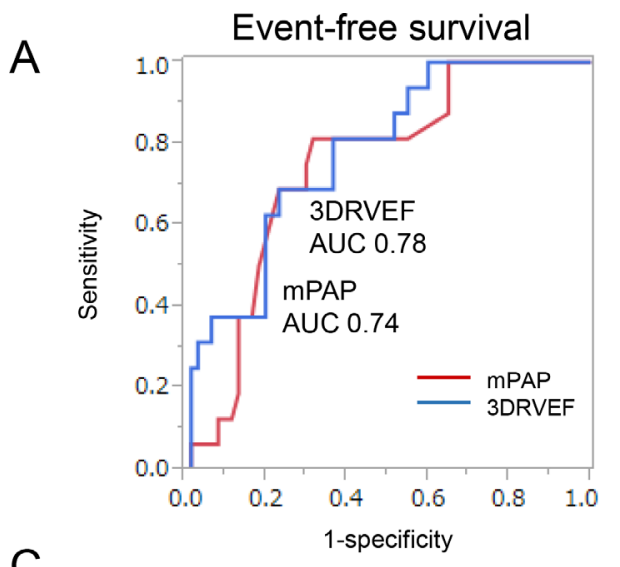

C

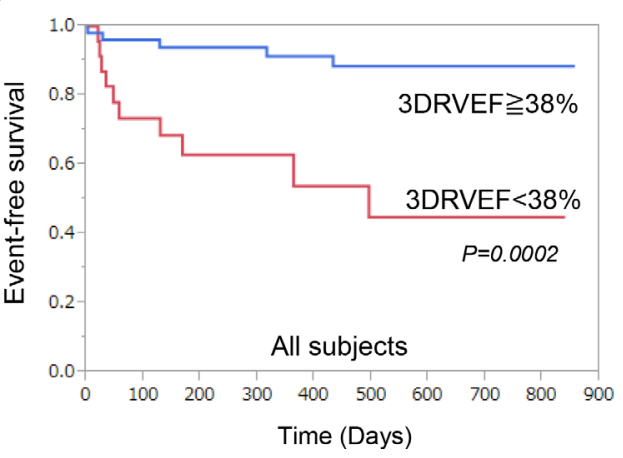

the RV-focused apical four-chamber view (RV 4CV), and then RVFAC was calculated. TAPSE was measured at the RV 4CV by achieving proper orientation for M-mode measures. RIMP was determined as the sum of the isovolumic contraction time and isovolumic relaxation time divided by ejection time. RAP was estimated based on the diameter of the inferior vena cava (IVC) from the long-axis subcostal view and its phasic response to respiration. RAP was estimated to be $3 \mathrm{mmHg}$ if the IVC was $<21 \mathrm{~mm}$ in diameter and collapsed by $>50 \%$ at the junction of the hepatic veins with a sniff, or $15 \mathrm{mmHg}$ if the IVC was $>21 \mathrm{~mm}$ in diameter and collapsed by $<50 \%$ with a sniff. Indeterminate cases in which the IVC diameter and collapse did not fit this paradigm were assigned an intermediate RA $P$ value of $8 \mathrm{mmHg}$. The peak tricuspid regurgitation (TR) velocity $(\mathrm{m} / \mathrm{s})$ was determined with continuous-wave Doppler using the highest velocity obtained from multiple views, and the TR pressure gradient (TRPG) was calculated as $4 \times$ (peak TR velocity) (2). RV systolic pressure was obtained by summing TRPG and RAP. The velocity, S', of RV was obtained by tissue Doppler imaging from RV-focused views at the lateral corner of the tricuspid annulus.

RV global and systolic function was also estimated by $2 \mathrm{DSTE}$. Gray scale imaging of the RV $4 \mathrm{CV}$ was
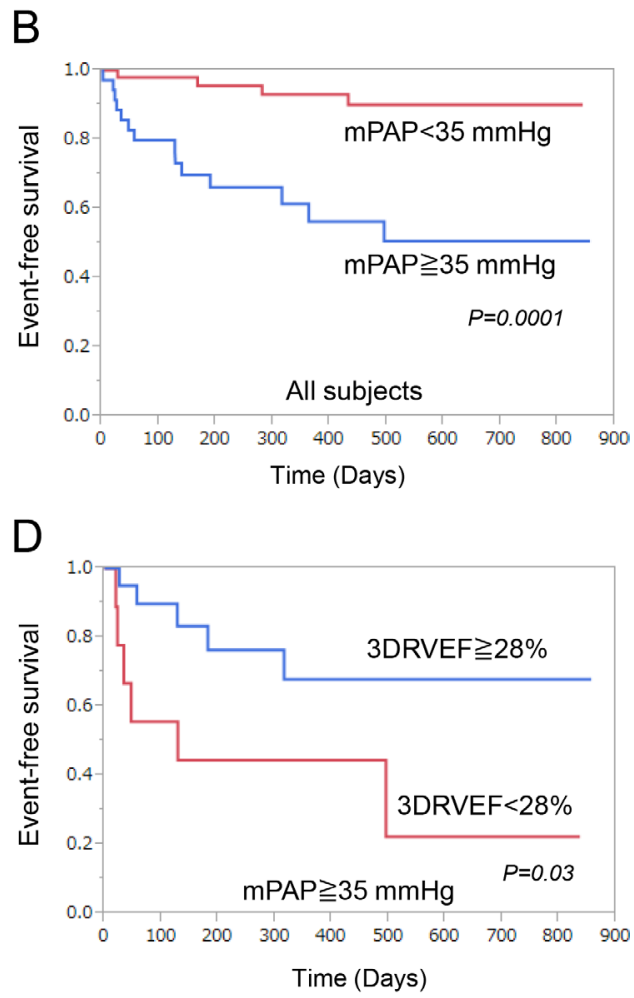

Figure 1: Correlation with clinical events. (A) Receiver-operating characteristic curve analysis revealed that 3DRVEF was superior to mPAP in predicting associated clinical events. (B) Event-free survival of patients with a mPAP $\geqq 35 \mathrm{mmHg}$ compared to patients with a mPAP $<35 \mathrm{mmHg}$ in all subjects. (C) Event-free survival of patients with a 3DRVEF $\geqq 38 \%$ compared to patients with a 3DRVEF $<38 \%$ in all subjects. (D) Event-free survival of patients with a $3 \mathrm{DRVEF} \geqq 28 \%$ compared to patients with a 3DRVEF $<28 \%$ in patients with severe $\mathrm{PAH}(\mathrm{mPAP}>35 \mathrm{mmHg}) .3 \mathrm{DRVEF}=$ three-dimensional right ventricular ejection fraction, $\mathrm{mPAP}=$ mean pulmonary arterial pressure. 
obtained with a frame rate of $40-80 \mathrm{~Hz}$, and recordings were processed with acoustic-tracking software (EchoPAC; GE Healthcare), allowing off-line semiautomated speckle-based strain analysis, as described previously [24]. Briefly, lines were first manually traced along the RV endocardium before an additional epicardial line was automatically generated by the software to create a region of interest. After manually adjusting the shape of the region of interest, the software divided the RV region into six segments and generated a longitudinal strain curve. We set the zero strain point as the time from the beginning of the QRS wave on the electrocardiogram, and measured negative peak strain during ventricular systole. In addition, RV dyssynchrony was quantified using the standard deviation of the heart rate-corrected intervals from QRS onset to peak systolic strain for the six segments (RV-SD6), as described previously [16, 25].

Three-dimensional echocardiographic images were obtained from the apical window with the patient in the same position as for 2D echocardiography, as described previously [24]. Briefly, echocardiographic images were stored digitally for offline analysis using TomTec 4D RV Function software (4D analysis; TomTec, Munich, Germany). Within the 3D data set, three orthogonal main cut planes were selected to define the end-diastolic and end-systolic frames within the sequence, as well as several landmarks. On the basis of the initial view adjustment and landmarks, the program automatically provided $4 \mathrm{CV}$, sagittal, and coronal RV views. RV end-diastolic volume, RV end-systolic volume, and RVEF were measured from each $3 \mathrm{D}$ echocardiographic data set.

The intra- and inter-observer reproducibility of strain parameters was shown to be acceptable. The intra- and inter-observer variabilities for 3DRVEF were $1.4 \pm 3.5 \%$ (95\% CI: -5.6 to 8.4$)$, and $1.5 \pm 3.8 \%(95 \%$ CI: -6.3 to 9.2$)$, respectively.

\section{Definitions of clinical outcomes}

Long-term unfavorable outcome events were prespecified as primary end points of death, hospitalization, or intervention including PEA or BPA for deteriorating right-sided heart failure. Mean follow-up was 423 days.

\section{Statistical analysis}

Quantitative results are expressed as mean \pm SD. Two-group comparisons were performed with unpaired Student $t$ tests for means if the data were normally distributed or with Wilcoxon rank-sum tests if the data were not normally distributed. Chi-square or Fisher exact tests were used to analyze the categorical data. Linear regression analysis was used to correlate two parameters. We calculated the cumulative incidence of events using the Kaplan-Meier method and compared the two curves with a log-rank test. We used Cox proportional hazards models to estimate hazard ratio (HR) for cardiovascular events and 95\% confidence interval. A multivariate-Cox proportional hazard model was also developed using stepwise regression by selecting those variables that were significant upon univariate analysis. Intra-observer variability and inter-observer variability of 3DTTE and RV longitudinal strain were calculated by a blinded repeat analysis of 20-25 randomly chosen patients. Intraobserver variability was performed 3 months after the first reading to avoid recall bias. Reproducibility was assessed by coefficient of variation. All statistical analyses were performed using JMP 11.0 software (SAS Institute, Cary, $\mathrm{NC}, \mathrm{USA})$.

\section{CONCLUSIONS}

RVEF measured by 3D echocardiography could be a useful parameter for noninvasively assessing RV hemodynamics and predicting clinical outcomes in patients with PAH.

\section{ACKNOWLEDGMENTS AND FUNDING}

The authors thank Ms Makiko Dan, Kumiko Abe, Yasuko Hatori, Akemi Okamoto, Makiko Kondo, and Mai Iwao for technical assistance.

\section{CONFLICTS OF INTEREST}

The authors declare no conflicts of interest.

\section{REFERENCES}

1. Kawut SM, Al-Naamani N, Agerstrand C, Rosenzweig EB, Rowan C, Barst RJ, Bergmann S, Horn EM. Determinants of right ventricular ejection fraction in pulmonary arterial hypertension. Chest. 2009; 135:752-759.

2. Anavekar NS, Skali H, Bourgoun M, Ghali JK, Kober L, Maggioni AP, McMurray JJ, Velazquez E, Califf R, Pfeffer MA, Solomon SD. Usefulness of right ventricular fractional area change to predict death, heart failure, and stroke following myocardial infarction (from the VALIANT ECHO Study). Am J Cardiol. 2008; 101:607-612.

3. Badagliacca R, Poscia R, Pezzuto B, Papa S, Gambardella C, Francone M, Mezzapesa M, Nocioni M, Nona A, Rosati R, Sciomer S, Fedele F, Dario Vizza C. Right ventricular dyssynchrony in idiopathic pulmonary arterial hypertension: determinants and impact on pump function. J Heart Lung Transplant. 2015; 34:381-389.

4. Badesch DB, Champion HC, Sanchez MA, Hoeper MM, Loyd JE, Manes A, McGoon M, Naeije R, Olschewski H, Oudiz RJ, Torbicki A. Diagnosis and assessment of pulmonary arterial hypertension. J Am Coll Cardiol. 2009; 54:S55-66.

5. Baggen VJ, Leiner T, Post MC, van Dijk AP, RoosHesselink JW, Boersma E, Habets J, Sieswerda GT. Cardiac 
magnetic resonance findings predicting mortality in patients with pulmonary arterial hypertension: a systematic review and meta-analysis. Eur Radiol. 2016.

6. Kato S, Sekine A, Kusakawa Y, Ogura T, Futaki M, Iwasawa T, Kirigaya H, Gyotoku D, Iinuma N, Iguchi K, Nakachi T, Fukui K, Kimura K, et al. Prognostic value of cardiovascular magnetic resonance derived right ventricular function in patients with interstitial lung disease. J Cardiovasc Magn Reson. 2015; 17:10.

7. Torbicki A. Cardiac magnetic resonance in pulmonary arterial hypertension: a step in the right direction. Eur Heart J. 2007; 28:1187-1189.

8. van Wolferen SA, Marcus JT, Boonstra A, Marques KM, Bronzwaer JG, Spreeuwenberg MD, Postmus PE, VonkNoordegraaf A. Prognostic value of right ventricular mass, volume, and function in idiopathic pulmonary arterial hypertension. Eur Heart J. 2007; 28:1250-1257.

9. Badagliacca R, Poscia R, Pezzuto B, Nocioni M, Mezzapesa M, Francone M, Giannetta E, Papa S, Gambardella C, Sciomer S, Volterrani M, Fedele F, Dario Vizza C. Right ventricular remodeling in idiopathic pulmonary arterial hypertension: adaptive versus maladaptive morphology. J Heart Lung Transplant. 2015; 34:395-403.

10. Brent BN, Berger HJ, Matthay RA, Mahler D, Pytlik L, Zaret BL. Physiologic correlates of right ventricular ejection fraction in chronic obstructive pulmonary disease: a combined radionuclide and hemodynamic study. Am J Cardiol. 1982; 50:255-262.

11. Burger W, Jockwig B, Rucker G, Kober G. Influence of right ventricular pre- and afterload on right ventricular ejection fraction and preload recruitable stroke work relation. Clin Physiol. 2001; 21:85-92.

12. Dubin AM, Janousek J, Rhee E, Strieper MJ, Cecchin F, Law IH, Shannon KM, Temple J, Rosenthal E, Zimmerman FJ, Davis A, Karpawich PP, Al Ahmad A, et al. Resynchronization therapy in pediatric and congenital heart disease patients: an international multicenter study. J Am Coll Cardiol. 2005; 46:2277-2283.

13. Lang RM, Badano LP, Mor-Avi V, Afilalo J, Armstrong A, Ernande L, Flachskampf FA, Foster E, Goldstein SA, Kuznetsova T, Lancellotti P, Muraru D, Picard MH, et al. Recommendations for cardiac chamber quantification by echocardiography in adults: an update from the American Society of Echocardiography and the European Association of Cardiovascular Imaging. J Am Soc Echocardiogr. 2015; 28:1-39 e14.

14. Gopal DM, Doldt B, Finch K, Simms RW, Farber HW, Gokce N. Relation of novel echocardiographic measures to invasive hemodynamic assessment in sclerodermaassociated pulmonary arterial hypertension. Arthritis Care Res (Hoboken). 2014; 66:1386-1394.

15. Mathai SC, Sibley CT, Forfia PR, Mudd JO, Fisher MR, Tedford RJ, Lechtzin N, Boyce D, Hummers LK, Housten T, Zaiman AL, Girgis RE, Hassoun PM. Tricuspid annular plane systolic excursion is a robust outcome measure in systemic sclerosis-associated pulmonary arterial hypertension. J Rheumatol. 2011; 38:2410-2418.
16. Yu CM, Lin H, Zhang Q, Sanderson JE. High prevalence of left ventricular systolic and diastolic asynchrony in patients with congestive heart failure and normal QRS duration. Heart. 2003; 89:54-60.

17. Sachdev A, Villarraga HR, Frantz RP, McGoon MD, Hsiao JF, Maalouf JF, Ammash NM, McCully RB, Miller FA, Pellikka PA, Oh JK, Kane GC. Right ventricular strain for prediction of survival in patients with pulmonary arterial hypertension. Chest. 2011; 139:1299-1309.

18. Ryo K, Goda A, Onishi T, Delgado-Montero A, Tayal B, Champion HC, Simon MA, Mathier MA, Gladwin MT and Gorcsan J, 3rd. Characterization of right ventricular remodeling in pulmonary hypertension associated with patient outcomes by 3-dimensional wall motion tracking echocardiography. Circ Cardiovasc Imaging. 2015; 8.

19. Miura Y, Fukumoto Y, Sugimura K, Oikawa M, Nakano M, Tatebe S, Miyamichi S, Satoh K, Shimokawa H. Identification of New Prognostic Factors of Pulmonary Hypertension. Circulation Journal. 2010; 74:1965-1971.

20. Badagliacca R, Reali M, Poscia R, Pezzuto B, Papa S, Mezzapesa M, Nocioni M, Valli G, Giannetta E, Sciomer S, Iacoboni C, Fedele F, Vizza CD. Right Intraventricular Dyssynchrony in Idiopathic, Heritable, and AnorexigenInduced Pulmonary Arterial Hypertension: Clinical Impact and Reversibility. JACC Cardiovasc Imaging. 2015; 8:642-652.

21. van de Veerdonk MC, Kind T, Marcus JT, Mauritz GJ, Heymans MW, Bogaard HJ, Boonstra A, Marques KM, Westerhof N, Vonk-Noordegraaf A. Progressive right ventricular dysfunction in patients with pulmonary arterial hypertension responding to therapy. J Am Coll Cardiol. 2011; 58:2511-2519.

22. van de Veerdonk MC, Marcus JT, Westerhof N, de Man FS, Boonstra A, Heymans MW, Bogaard HJ, Vonk Noordegraaf A. Signs of right ventricular deterioration in clinically stable patients with pulmonary arterial hypertension. Chest. 2015; 147:1063-1071.

23. Freed BH, Tsang W, Bhave NM, Patel AR, Weinert L, Yamat M, Vicedo BM, Dill K, Mor-Avi V, GombergMaitland M, Lang RM. Right ventricular strain in pulmonary arterial hypertension: a 2D echocardiography and cardiac magnetic resonance study. Echocardiography. 2015; 32:257-263.

24. Tsugu T, Murata M, Kawakami T, Yasuda R, Tokuda H, Minakata Y, Tamura Y, Kataoka M, Hayashida K, Tsuruta H, Maekawa Y, Inoue S, Fukuda K. Significance of echocardiographic assessment for right ventricular function after balloon pulmonary angioplasty in patients with chronic thromboembolic induced pulmonary hypertension. Am J Cardiol. 2015; 115:256-261.

25. Conca C, Faletra FF, Miyazaki C, Oh J, Mantovani A, Klersy C, Sorgente A, Pedrazzini GB, Pasotti E, Moccetti T, Auricchio A. Echocardiographic parameters of mechanical synchrony in healthy individuals. Am J Cardiol. 2009; 103:136-142. 